International Journal of Basic and Applied Sciences, 6 (4) (2017) $73-76$
International Journal of Basic and Applied Sciences
Website: www.sciencepubco.com/index.php/IJBAS
doi: $10.14419 /$ ijbas.v6i4.7969
Research paper

\title{
A dynamic economic model of criminal activity in the criminal law
}

\author{
Fatemeh Seddighi Chaharborj ${ }^{1}$, Babak Pourghahramani ${ }^{1}$ *, Sarkhosh Seddighi Chaharborj ${ }^{2,} 3$ * \\ ${ }^{1}$ Department of Law, Islamic Azad University Maragheh, Iran \\ ${ }^{2}$ Department of Mathematics, Islamic Azad University, Bushehr Branch, Bushehr, Iran \\ ${ }^{3}$ Department of Mathematics and Statistics, Carleton University, Ottawa, \\ *Corresponding author E-mail: b.pourghahramani@yahoo.com, sseddighi2014@yahoo.com.my
}

\begin{abstract}
Poverty and crime are two major problem areas. The economic theory of crime shows a direct correlation between poverty and crime. In this study, we propose a model that shows the correlation between poverty and crime. Then we obtain the dynamic system of the proposed model. In the next step, we will compute the reproductive number by finding the maximum eigenvalue of Jacobian matrix to study of stability and un-stability of the presented model.
\end{abstract}

Keywords: Criminal Activity; Criminal Law; Economic Model; Dynamic System; Reproductive Number; Jacobian Matrix.

\section{Introduction}

Economic theory of crime has direct association between poverty and crimes. The model of this study attempts to evaluate the dynamics of poverty system-crime via stability analysis of ODE system as performed to identify economical strategies to fight against crime in big cities.

There is a direct association between poverty and crimes $[1,8]$. Becker's economic theory about crime (1968) states that people choose delinquency when crime committing is lower than achieved benefits. Thus, those living in poverty can commit the crime than public population $[1,2]$. Delinquency is defined as theft, stealing [1-3]. In the paper of Becker, statistical and economic analysis is used to determine optimal control of crime [1].

Here, we test a dynamic system of presented model and achieve realistic and dynamic solutions of this question [4-7]. We observe that from economic aspects, arresting each prisoner creates a higher load in society compared with the crime itself. We will compute the reproductive number by finding the maximum eigenvalue of Jacobian matrix to study of stability and un-stability of the presented model. Finally, the goal is to reduce crime as total costs of crime control and costs of remained crimes are less than total costs of crime under the existing condition.

\section{Mathematical modeling}

Figure (1) indicates the relationship between non-impoverished and poor people, jailed and rehabilitated. Here, we have non-impoverished class $\mathrm{N}$, the poverty class $\mathrm{P}$, the criminal class $\mathrm{C}$, the jailed class $\mathrm{J}$ and the recovered class $\mathrm{R}$ and $T=N+P+C+J+R$ for total people in society. Ordinary differential equations or dynamic systems for Figure (1) are shown as followings:

$$
\left\{\begin{array}{l}
\frac{d N}{d t}=\mu T-\left(\mu+\alpha_{1}\right) N=F_{1}, \\
\frac{d P}{d t}=\alpha_{1} N-\left(\mu+\alpha_{2} \frac{C}{T}+\alpha_{5}\right) P=F_{2}, \\
\frac{d C}{d t}=\alpha_{2} \frac{C}{T} P+\alpha_{2} \alpha_{6} \frac{C}{T} R-\left(\mu+\alpha_{3}\right) C=F_{3}, \\
\frac{d J}{d t}=\alpha_{3}+\alpha_{4} J-\left(\mu+\alpha_{4}\right) J=F_{4}, \\
\frac{d R}{d t}=\alpha_{5} P+\alpha_{4} J-\left(\mu-\alpha_{2} \alpha_{6} \frac{C}{T}\right) R=F_{5} .
\end{array}\right.
$$

Here, $\frac{d N}{d t}=0, \frac{d P}{d t}=0, \frac{d C}{d t}=0, \frac{d J}{d t}=0$, and $\frac{d R}{d t}=0$ denote the speed of increase of non-impoverished, poor, criminal, jailed and recovered. Let $\mu$ denotes death speed, $\sigma$ denotes the rate of the flow from the non-impoverished class $\mathrm{N}$ to the impoverished class P. $\alpha_{2}$ indicates transmission probability (for example, probability speed of transferring poor people to criminals or recovered people to criminals), $\alpha_{3}$ denotes speed of jailing of criminals, $\alpha_{4}$ is releasing of jailed from jail, $\alpha_{5}$ denotes speed of recovery of impoverished without committing crime, $0 \leq \alpha_{6} \leq 1$ is transmission coefficient of recovered people to criminal people and $\frac{C}{T}$ is probability of criminals to total people in society. 


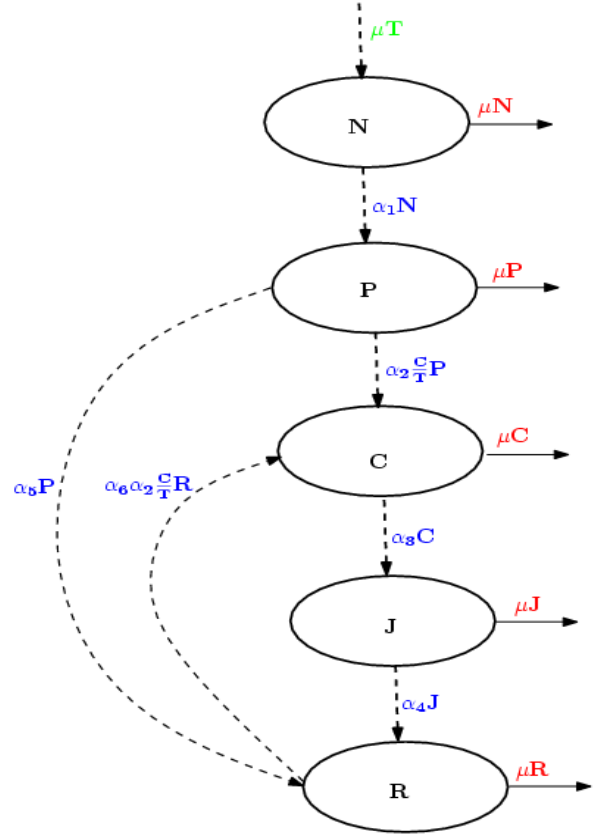

Fig. 2: Flowchart of Transition Stages of People in Modelling the Relationship between Poverty and Crime.

As shown in Figure (1), in order that recovered or healthy people commit crime, it depends upon the fact that healthy person visits the criminal on which probability and it is regarding the ratio of criminal to total people, the probability of criminals and probability of transferring healthy people to commit crime. In order that a poor person commits crime, it depends upon the probability of criminals and transmission speed of impoverished to criminals.

Important discussions of great important in such modellings include reproductive number as obtained via finding the greatest eigenvalue and it is system stability points or model. To find stability points, $\frac{d N}{d t}=0, \frac{d P}{d t}=0, \frac{d C}{d t}=0, \frac{d J}{d t}=0$, and $\frac{d R}{d t}=0$ are applied. The stability zone is plotted via reproductive number to define all stability and convergence points and all instability and divergence points.

\section{Reproductive number}

Reproductive Number is denoted by $\Re$ and to find it, derivative and Jacobian methods are used. In derivative method, ascending and descending states are used for stability and non-stability of required system. In Jacobian method, $|J-\lambda I|=0$ is used and greatest eigenvalue is found. Here, $\mathrm{J}$ is Jacobian matrix and $\lambda$ is eigenvalue and $\mathrm{I}$ is elementary matrix.

\subsection{Direct method to find reproductive number}

Note 1: One of the simple methods to find reproductive value $\Re=$ $\Re_{P}+\Re_{R}$, is solving equations $F_{i}=0,(i=1, \ldots, 5)$ as followings: At first, by using the equations $F_{i}=0,(i=1,2,3), \mathfrak{R}_{P}$ can be found as follows:

$$
\begin{gathered}
\mu T-\left(\mu+\alpha_{1}\right) N=0 \Longrightarrow N=\left(\frac{\mu}{\mu+\alpha_{1}}\right) T, \\
\alpha_{1} N-\left(\mu+\alpha_{2} \frac{C}{T}+\alpha_{5}\right) P=0 \Longrightarrow P=\left(\frac{\alpha_{1}}{\mu+\alpha_{5}}\right) N, \\
\alpha_{2} \frac{C}{T} P+\alpha_{2} \alpha_{6} \frac{C}{T} R-\left(\mu+\alpha_{3}\right) C=0 \Longrightarrow C=\left(\frac{\alpha_{2}}{\mu+\alpha_{3}}\right) P,
\end{gathered}
$$

Therefore, based on this solution, first reproductive, can be written as:

$$
\mathfrak{R}_{P}=C=\left(\frac{\alpha_{2}}{\mu+\alpha_{3}}\right) \times\left(\frac{\alpha_{1}}{\mu+\alpha_{5}}\right) \times\left(\frac{\mu}{\mu+\alpha_{1}}\right) .
$$

By using the equations $F_{i}=0,(i=3,4,5), \mathfrak{R}_{R}$ can be found as follows:

$$
\begin{gathered}
\alpha_{1} N-\left(\mu+\alpha_{2} \frac{C}{T}+\alpha_{5}\right) P=0 \Rightarrow P=\left(\frac{\alpha_{1}}{\mu+\alpha_{5}}\right) N, \\
\alpha_{2} \frac{C}{T} P+\alpha_{2} \alpha_{6} \frac{C}{T} R-\left(\mu+\alpha_{3}\right) C=0 \Rightarrow C=\left(\frac{\alpha_{2} \alpha_{6}}{\mu+\alpha_{3}}\right) P, \\
\alpha_{5} P+\alpha_{4} J-\left(\mu-\alpha_{2} \alpha_{6} \frac{C}{T}\right) R=0 \Rightarrow J=\left(\frac{\alpha_{3}}{\mu+\alpha_{4}}\right) C,
\end{gathered}
$$

Therefore, based on this solution, second reproductive, can be written as:

$$
\mathfrak{R}_{P}=J=\left(\frac{\alpha_{2} \alpha_{6}}{\mu+\alpha_{3}}\right) \times\left(\frac{\alpha_{1}}{\mu+\alpha_{5}}\right) \times\left(\frac{\alpha_{3}}{\mu+\alpha_{4}}\right) .
$$

Now according definition $\Re=\Re_{P}+\Re_{R}$, general formula for the reproductive number $(\Re)$ can be written as follows:

$$
\begin{aligned}
\Re=\left(\frac{\alpha_{2}}{\mu+\alpha_{3}}\right) \times\left(\frac{\alpha_{1}}{\mu+\alpha_{5}}\right) \times\left(\frac{\mu}{\mu+\alpha_{1}}\right) & \\
& +\left(\frac{\alpha_{2} \alpha_{6}}{\mu+\alpha_{3}}\right) \times\left(\frac{\alpha_{1}}{\mu+\alpha_{5}}\right) \times\left(\frac{\alpha_{3}}{\mu+\alpha_{4}}\right) .
\end{aligned}
$$

\subsection{Jacobian method to find the reproductive number}

In this section, we try to find general formula for the reproductive number $(\mathfrak{R})$ by using the Jacobian method. At first Jacobian matrix of system (1) is formed and then the eigenvalues of this matrix are found and via greatest eigenvalue, reproductive number is found. We find the Jacobian matrix around the equilibrium point of system (1). To find the Jacobian matrix around the equilibrium point the below equations will be used:

$\left\{\begin{array}{l}F_{1}=\mu T-\left(\mu+\alpha_{1}\right) N, \\ F_{2}=\alpha_{1} N-\left(\mu+\alpha_{2} \frac{C}{T}+\alpha_{5}\right) P, \\ F_{3}=\alpha_{2} \frac{C}{T} P+\alpha_{2} \alpha_{6} \frac{C}{T} R-\left(\mu+\alpha_{3}\right) C, \\ F_{4}=\alpha_{3}+\alpha_{4} J-\left(\mu+\alpha_{4}\right) J, \\ F_{5}=\alpha_{5} P+\alpha_{4} J-\left(\mu-\alpha_{2} \alpha_{6} \frac{C}{T}\right) R .\end{array}\right.$

The Jacobian matrix for this equations can be defined as follows:

$$
J=\left[\begin{array}{lllll}
\frac{d F_{1}}{d N} & \frac{d F_{1}}{d P} & \frac{d F_{1}}{d C} & \frac{d F_{1}}{d J} & \frac{d F_{1}}{d R} \\
\frac{d F_{2}}{d N} & \frac{d F_{2}}{d P} & \frac{d F_{2}}{d C} & \frac{d F_{2}}{d J} & \frac{d F_{2}}{d R} \\
\frac{d F_{3}}{d N} & \frac{d F_{3}}{d P} & \frac{d F_{3}}{d C} & \frac{d F_{3}}{d J} & \frac{d F_{3}}{d R} \\
\frac{d F_{4}}{d N} & \frac{d F_{4}}{d P} & \frac{d F_{4}}{d C} & \frac{d F_{4}}{d J} & \frac{d F_{4}}{d R} \\
\frac{d F_{5}}{d N} & \frac{d F_{5}}{d P} & \frac{d F_{5}}{d C} & \frac{d F_{5}}{d J} & \frac{d F_{5}}{d R}
\end{array}\right]
$$

Eigenvalue of this matrix is found by the following equation easily: Note 1: If $\lambda_{\max }$ is the greatest eigenvalue of $A_{5 \times 5}$ matrix, then if $\lambda_{\max }>0$, the system is stable and if $\lambda_{\max }<0$, the system is unstable and if $\lambda_{\max }=0$, the system is under critical condition.

Finally, by using the greatest eigenvalue the reproductive number is found as follows:

$$
\Re=\Re_{P}+\Re_{R}
$$




$$
\begin{aligned}
& \mathfrak{R}_{P}=\left(\frac{\alpha_{2}}{\mu+\alpha_{3}}\right) \times\left(\frac{\alpha_{1}}{\mu+\alpha_{5}}\right) \times\left(\frac{\mu}{\mu+\alpha_{1}}\right), \\
& \mathfrak{R}_{R}=\left(\frac{\alpha_{2} \alpha_{6}}{\mu+\alpha_{3}}\right) \times\left(\frac{\alpha_{1}}{\mu+\alpha_{5}}\right) \times\left(\frac{\alpha_{3}}{\mu+\alpha_{4}}\right) .
\end{aligned}
$$

Factors $\frac{\alpha_{2}}{\mu+\alpha_{3}}$ and $\frac{\alpha_{2} \alpha_{6}}{\mu+\alpha_{3}}$ factors show the number of new delinquents of classes $\mathrm{P}, \mathrm{R}$ as produced by a delinquent during criminal periods, before being jailed. $\frac{\alpha_{1}}{\mu+\alpha_{5}}$ factor denotes the probability that a nonimpoverished person enters an impoverished class while $\frac{\mu}{\mu+\alpha_{1}}$ and $\frac{\alpha_{3}}{\mu+\alpha_{4}}$ respectively are the probabilities in which a person $\mathrm{P}$ is remained in class $\mathrm{p}$ or is transferred to class $\mathrm{R}$.

Note 2: If $\Re$ is reproductive number for matrix $A_{5 \times 5}$, then if $\Re>1$, the system is stable and if $\Re<1$, the system is instable and if $\Re=$ 1 , the system is under critical condition.

\section{Numerical study}

\subsection{Convergence study}

In this section we want to study convergence of solutions to freeequilibrium point. Initial values for this purpose have been used as follows:

$$
\begin{gathered}
N(0)=1000, P(0)=100, C(0)=50, J(0)=25, R(0)=0 \\
\alpha_{1}=0.4, \alpha_{2}=0.1, \alpha_{3}=0.2, \alpha_{4}=0.15, \alpha_{5}=0.3, \alpha_{6}=0.25, \gamma \\
=0.05
\end{gathered}
$$

Figure (2-5) are showing the convergence of solutions when time goes to infinity. This points are free-equilibrium points. Figure (2) shows convergence of poverty and criminal solutions to free-equilibrium point as $(149.21,0)$. Convergence of poverty and jailed solutions to the free-equilibrium point $(149.21,0)$ is presented in figure (3). Figure (4) indicates convergence of poverty and recovery solutions to $(149.21,894.24)$ as free-equilibrium point and figure (5) displays convergence of criminal and recovery solutions to point $(0,894.24)$ as free-equilibrium point.

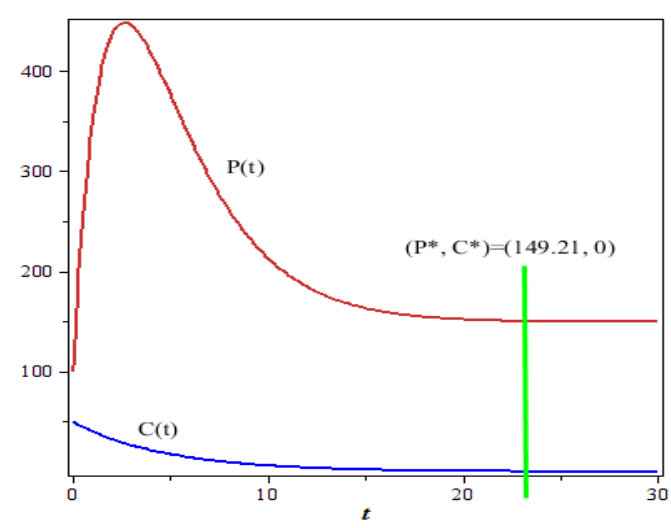

Fig. 2: Convergence of Poverty and Criminal Solutions to Point (149.21, 0).

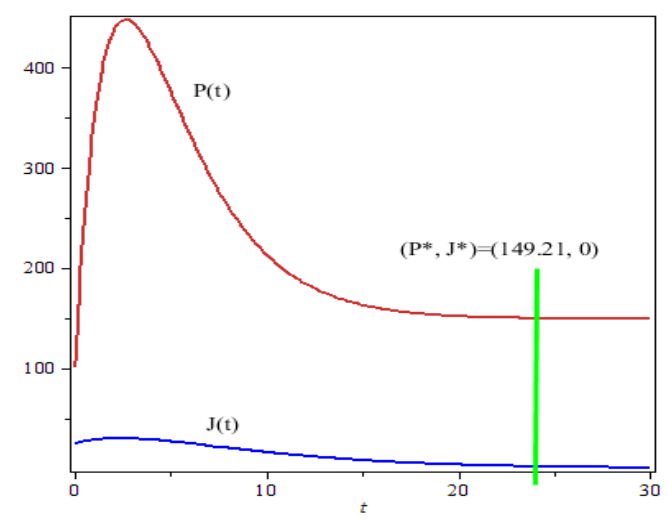

Fig. 3: Convergence of Poverty and Jailed Solutions to Point (149.21, 0).

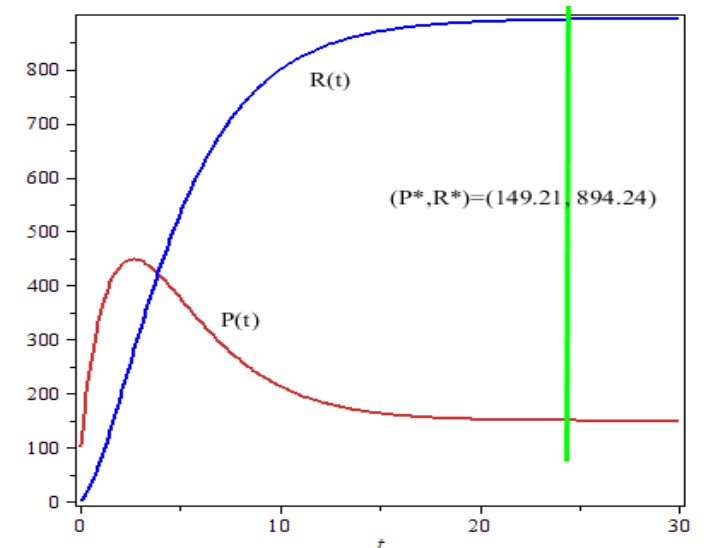

Fig. 4: Convergence of Poverty and Recovery Solutions to Point (149.21, 894.24).

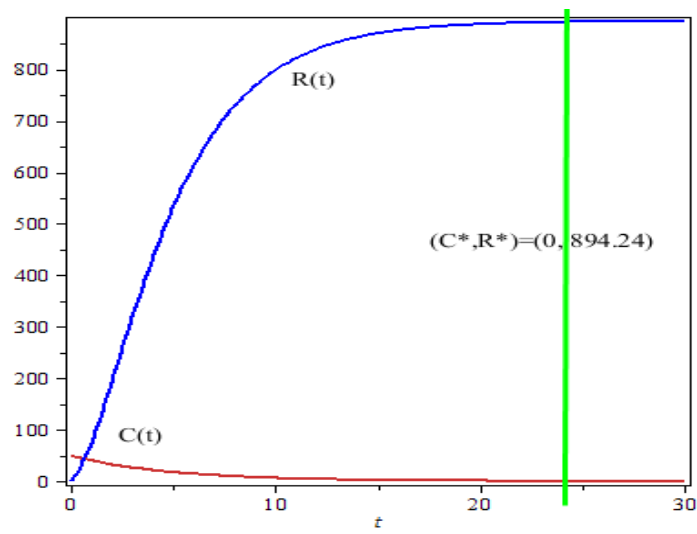

Fig. 5: Convergence of Criminal and Recovery Solutions to Point $(0$, 894.24).

Role of imprisoning of criminal people in the sustainability and constancy of a community

\subsection{Role of imprisoning of criminal people in the sustain- ability and constancy of a community}

In this section we study the role of imprisoning of criminal people in the sustainability and constancy of a community. Initial values for this purpose have been used as follows:

$$
\begin{gathered}
N(0)=20000, P(0)=5000, C(0)=200, J(0)=50, R(0)=0, \\
\begin{array}{c}
\alpha_{1}=0.6, \alpha_{2}=0.7,0 \leq \alpha_{3} \leq 1, \alpha_{4}=0.6, \alpha_{5}=0.8, \alpha_{6}=0.7, \mu \\
=0.1 .
\end{array}
\end{gathered}
$$

Figures (6-9) are showing the poverty, criminal, jailed and recovered population as function of parameter $\alpha_{3}$. Here $\alpha_{3}$ defines speed of peoples from jailing class to criminal class. Figure (6) shows the poverty population as a function of $\alpha_{3}$; in this figure with increasing $\alpha_{3}$ from 0 to 1 , poverty populations going to decrease. Maximum values for all curves are between times 0 to 10 and after time 10 all these looks stable and constant to free-equilibrium points. The criminal population as function of parameter $\alpha_{3}$ has been shown in figure (7). As seen from figure (7), with increasing parameter $\alpha_{3}$ from 0 to 1 criminal population decreases and criminal population with more rapidly is going to zero. Figure (8) indicates the jailed population as function of parameter $\alpha_{3}$. In this figure with increasing parameter $\alpha_{3}$ from 0 to 1 , the jailed population increases. This shows that the police have done better its role in the jailing criminals' population. The recovered population as function of parameter 
$\alpha_{3}$ has been shown in figure (9). Figure (9) is showing that with increasing parameter $\alpha_{3}$ from 0 to 1 , also recovered population increases.

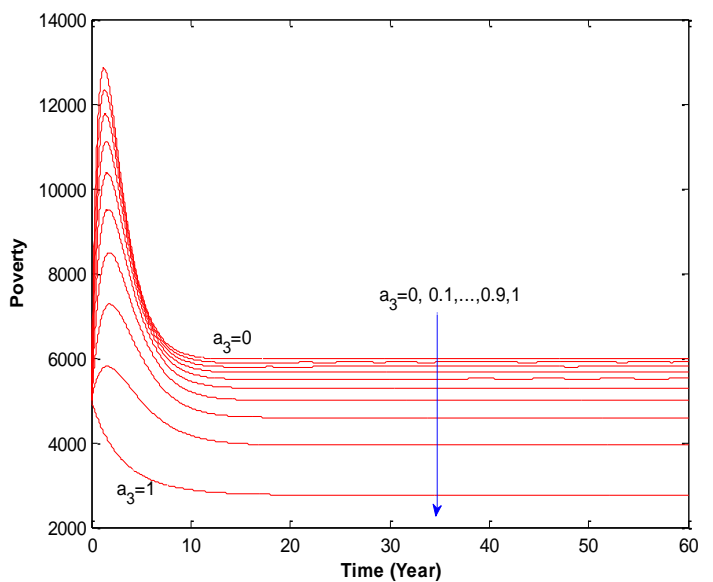

Fig. 6: Poverty Population as Function of Parameter $\alpha_{3}\left(\alpha_{3}\right.$ : Speed of Jailing of Criminals).

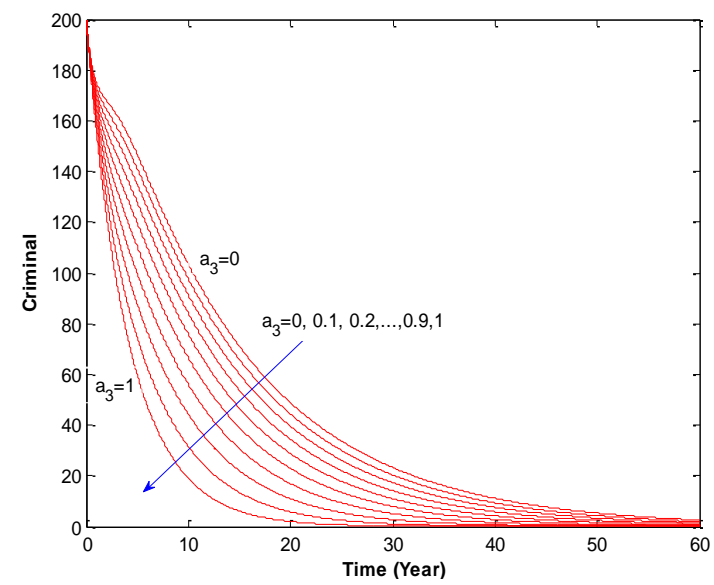

Fig. 7: Criminal Population as Function of Parameter $\alpha_{3}\left(\alpha_{3}\right.$ : Speed of Jailing of Criminals).

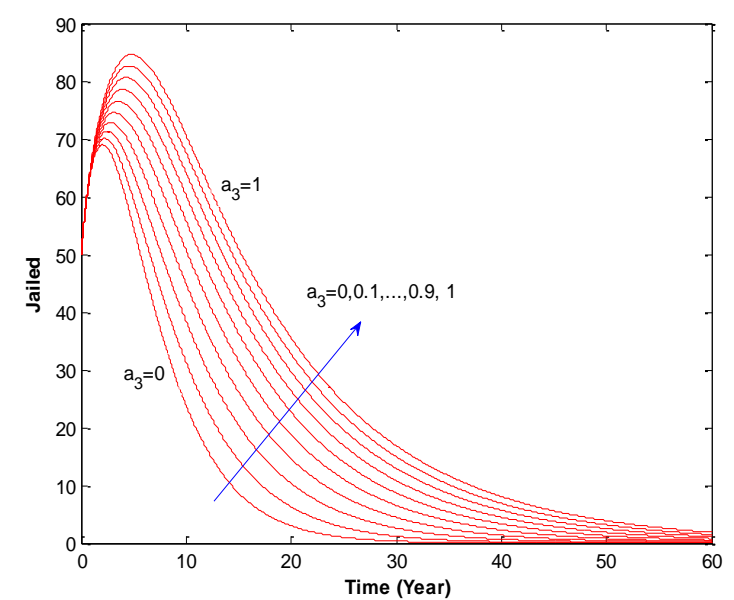

Fig. 8: Jailed Population as Function of Parameter $\alpha_{3}\left(\alpha_{3}\right.$ : Speed of Jailing of Criminals).

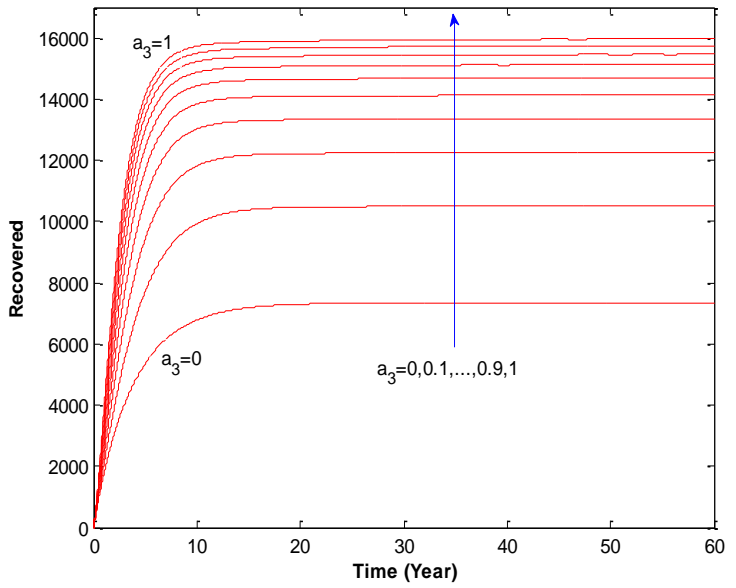

Fig. 9: Recovered Population as Function of Parameter $\alpha_{3}\left(\alpha_{3}\right.$ : Speed of Jailing of Criminals).

\section{Conclusion}

The economic theory of poverty and crime has been modeled as dynamic systems. Study of the proposed dynamic system shows the correlation between poverty and crime. Important parameters of the presented method have been appeared in the computed reproductive. This means that reproductive number is playing an important role in dynamic systems. When the reproductive number is greater than, equal or less than 1; model is un-stable, critical or stable, respectively. In stable case, the poverty, criminal and jailed populations will have reached to zero, and this will show an ideal society. In the next, we study the role of imprisoning of criminal people in the sustainability and constancy of a community. We showed that the police important role in controlling the societies and can be more helpful in keeping society stable. This will reduce crime as total costs of crime control.

\section{References}

[1] Baker 2007, A Dynamic Model of Crime and Punishment, new York. Ny, 10021.

[2] Chow, C., Gutkin, B., Hansel, D., Meunier, C. \& Dalibard, J.(editors) (2004) Methods and Models in Neurophysics. Session LXXX, Lecture Notes of the Les Houches Summer School 2003, Elsevier.

[3] Gordon, M. B. (2010). A random walk in the literature on criminality: A partial and critical view on some statistical analyses and modelling approaches. European Journal of Applied Mathematics, 21(4-5), 283-306. https://doi.org/10.1017/S0956792510000069.

[4] Sarkhosh, S. Ch., Abu Bakar, M. R., \& Ebadian, A. (2010). Disease transmission MSEIR model with individuals traveling between patches i and i+ 1. Journal of Applied Mathematics and Informatics, 28(5-6), 1073-1088.

[5] Sarkhosh, S. Ch., Bakar, M., Fudziah, I., Akma, I., Malik, A., \& Alli, V. (2010). Behavior stability in two SIR-style models for HIV. Int. Journal of Math Analysis, 4(9), 427-434.

[6] Sarkhosh, S. Ch., Fudziah, I., Bakar, M. A., Chaharborj, R. S., Majid, Z. A., \& Ahmad, A. G. B. (2013). The use of generation stochastic models to study an epidemic disease. Advances in Difference Equations, 2013(1), 1-9.

[7] Sarkhosh, S. Ch., Mohd Rizam, A. B., \& Fudziah, I. (2012). Study of stochastic systems for epidemic disease models. In International Journal of Modern Physics: Conference Series (Vol. 9, pp. 373-379). World Scientific Publishing Company.

[8] Zhao, H., Feng, Z., \& Castillo-Chavez, C. (2014). The dynamics of poverty and crime. Journal of Shanghai Normal University (Natural Sciences. Mathematics). 